\title{
A Measure Theoretical Approach for Path Planning Problem of Nonlinear Control Systems
}

\author{
Amin Jajarmi ${ }^{1}$, Hamidreza Ramezanpour ${ }^{2}$, Mohammad Dehghan Nayyeri ${ }^{3}$, Ali Vahidian Kamyad ${ }^{3}$ \\ ${ }^{1}$ Department of Electrical Engineering, Ferdowsi University of Mashhad, Mashhad, Iran \\ ${ }^{2}$ Department of Nuclear Engineering and Physics, AmirKabir University of Technology, Tehran, Iran \\ ${ }^{3}$ Department of Applied Mathematics, Ferdowsi University of Mashhad, Mashhad, Iran \\ E-mail:jajarmi@stu-mail.um.ac.ir \\ Received October 19, 2010; revised May 13, 2011; accepted May 15, 2011
}

\begin{abstract}
This paper presents a new approach to find an approximate solution for the nonlinear path planning problem. In this approach, first by defining a new formulation in the calculus of variations, an optimal control problem, equivalent to the original problem, is obtained. Then, a metamorphosis is performed in the space of problem by defining an injection from the set of admissible trajectory-control pairs in this space into the space of positive Radon measures. Using properties of Radon measures, the problem is changed to a measure-theoretical optimization problem. This problem is an infinite dimensional linear programming (LP), which is approximated by a finite dimensional LP. The solution of this LP is used to construct an approximate solution for the original path planning problem. Finally, a numerical example is included to verify the effectiveness of the proposed approach.
\end{abstract}

Keywords: Path Planning, Optimal Control, Measure Theory, Linear Programming

\section{Introduction}

In the control theory, the path planning problem finds an admissible control for steering the control system from an initial state to a desired final state in a certain finite time interval. This problem has been developed initially by the aerospace industries for trajectory modification of aircrafts and space vehicles [1]. Moreover, it is one of the most applicable control problems, especially in robot industrial and etc [2-4]. However, the inherent nonlinearity of practical systems presents a challenging path planning problem. For many systems, the conventional trial and error method can work quite well to find system schedules. But for more advanced ones, more accurate methodologies are needed. For instance, in [5] the problem of optimal path planning has been considered as a semi- infinite constrained optimization problem.

In the filed of path planning, many different solution methods have been developed [6]. Most of the conventional methods, such as road mapping [7] and potential field [8], have some weaknesses in common. In the road mapping method, which is probabilistic, the heuristic nature of path generation leads to the difficulty in characterizing the algorithm in terms of performance, com- plexity, and reliability [9]. Potential field path planning method has been appeared frequently in the literatures; however, it has been plagued with inherent limitations [10].

In [11] a chaotic genetic algorithm has been used to find the shortest path for a mobile robot to move in a static environment. Besides, in [12] a chaotic particle swarm optimization (PSO) algorithm with mutation operator has been employed in the path planning. But, since the path planning is a complex NP-hard problem, general particle swarm optimizer is slow in convergence and is easy to be trapped in local optima, especially in complex multi-apex search problem. In [13] a variational approach has been proposed for path planning in three dimensions by defining an energy integral over the path, using gradient flow on the defined energy, and evolving the entire path until a locally optimal steady state is reached. A mixed integer linear programming (MILP) method has also been proposed in $[14,15]$ which yields an optimization-based technique and performs quite well in specific instances. This method combines linear programming (LP) problem with the ability of constraining some subset of the state variables to be integers.

In the past few years, the idea of finding solutions of 
some problems by converting them to a suitable optimal control problem has received growing attentions. In [16-18] you can see some applications of this idea for solving a number of ordinary and partial differential equations. The path planning problem can also be converted to an optimal control problem by considering a suitable objective function. Therefore, optimal control concepts can be used which present a systematic approach to solve the problem. In [19], a new optimal control problem, equivalent to the path planning problem, has been obtained by defining a new formulation in the calculus of variations. Discretizing this new problem yields a nonlinear programming (NLP) which may have a large number of variables and a large number of constraints. To overcome this difficulty and reduce the computational complexity, an iterative algorithm has also been introduced in [19] in which a sequence of reduced order NLP's is solved instead of directly solving the large NLP obtained through the discretization. However, solving NLP problems is much more difficult than solving LP ones.

In [20] a suitable tool is introduced to obtain approximate solutions for optimal control problems using the concept of measure theory [21-23]. In this approach, to find an acceptable solution, only a LP problem should be solved. Therefore, the approach has the advantage that it sets aside completely the nonlinearity of the problem. Moreover, it does not depend on the convexity of objective function. Besides, it is practical for systems with too complicated nonlinear terms.

In this paper, using the concept of measure theory, a novel practical approach is proposed to approximate the solution of nonlinear path planning problem. The proposed approach in comparison with other numerical methods works well; especially it is practical and accurate enough for systems with too complicated nonlinear terms. Moreover, as the obtained control function is piecewise constant, it is suitable for switching systems. Besides, error is completely controllable and accuracy can be improved as fine as desired.

The paper is organized as follows. Section 2 defines the problem of path planning, and Section 3 proposes a new formulation for this problem. In Section 4, a metamorphosis is performed to convert the problem to an infinite-dimensional LP in measure space. Then, by introducing two stage approximations in Section 5, a finite-dimensional LP is obtained. In Section 6, an illustrative example is presented to verify the effectiveness of the proposed approach. Finally, conclusions are given in the last section.

\section{Definition}

Consider the following general form of nonlinear path planning problem:

$$
\begin{aligned}
& \dot{x}(t)=g(x(t), u(t), t) \\
& \text { s.t. } \\
& x(t) \in A, u(t) \in U \\
& x\left(t_{a}\right)=x_{a}, x\left(t_{b}\right)=x_{b} \\
& t \in J=\left[t_{a}, t_{b}\right]
\end{aligned}
$$

where $x(\cdot): J \rightarrow R^{n}$ is the state trajectory which is assumed to be absolutely continuous on $J$ and be constrained to stay in the compact set $A \subseteq R^{n}$,

$u(\cdot): J \rightarrow R^{m}$ is the control function which is a bounded measurable function on $J$ and takes its values in the compact set $U \subseteq R^{m}, g(\cdot): \Omega \rightarrow V$ is a continuous function where $V \subseteq R^{n}$ is a compact set and $\Omega=A \times U \times J, x_{a} \in A$ and $x_{b} \in A$ are the initial and final states, respectively. In addition, it is assumed that $x(t)$ satisfies the differential equation of system, almost everywhere on $J$. Path planning problem is the problem of guiding control system from the known initial state $x_{a}$ at $t_{0}=t_{a}$ to the given final state $x_{b}$ at $t_{f}=t_{b}$.

Remark 1.1. Under the above-mentioned assumptions, the nonlinear system in (1) without the final condition $x\left(t_{b}\right)=x_{b}$ has a unique solution $x(t)$ for every bounded measurable control function $u(t)$ [24]. In this situation, under assumption that $\frac{\partial g}{\partial x}$ be continuous on $\Omega$, the solution of system obtained from a piecewise continuous control function $u(t)$ is piecewise $C^{1}$ function, i.e. $x(t)$ is continuous piecewise differentiable and has piecewise continuous derivative [24].

\section{New Formulation}

In [25], there is a new formulation for the path planning problem of linear time-varying systems. Here, that formulation is extended for the nonlinear system in (1) by defining a function $F$ as follows:

$$
\left\{\begin{array}{l}
F: R^{2 n+m+1} \rightarrow R^{+} \\
F(x(t), \dot{x}(t), u(t), t) \triangleq\|\dot{x}(t)-g(x(t), u(t), t)\|
\end{array}\right.
$$

where $\|\cdot\|$ is a suitable continuous norm on $R^{n}$ and $R^{+}=[0, \infty)$. Now the following variational problem can be defined:

$$
\begin{aligned}
& \min \int_{J} F(x(t), \dot{x}(t), u(t), t) \mathrm{d} t \\
& \text { s.t. } \\
& x(t) \in A, u(t) \in U \\
& x\left(t_{a}\right)=x_{a}, x\left(t_{b}\right)=x_{b}, t \in J
\end{aligned}
$$

where $A, U$, and $J$ are as before. 
Problem (3) is equivalent to the original problem (1) as the following theorem states:

Theorem 3.1. Problem (1) has a solution if and only if problem (3) has an optimal solution with the corresponding zero optimal objective value.

Proof. The "only if" part is obvious. For the "if" part let $\left(x^{*}(\cdot), u^{*}(\cdot)\right)$ be the optimal solution of problem (3) with corresponding zero optimal objective value, that is $\int_{J} F\left(x^{*}(t), \dot{x}^{*}(t), u^{*}(t), t\right) \mathrm{d} t=0$. Since the integrand is nonnegative real-valued function, we have $F\left(x^{*}(t), \dot{x}^{*}(t), u^{*}(t), t\right)=0$ almost everywhere on $J$, and so $\dot{x}^{*}(t)-g\left(x^{*}(t), u^{*}(t), t\right)=0$ almost everywhere on $J$, and $x^{*}\left(t_{a}\right)=x_{a}$ and $x^{*}\left(t_{b}\right)=x_{b}$. As $x^{*}(t)=x^{*}\left(t_{a}\right)+\int_{t_{a}}^{t} \dot{x}^{*}(s) \mathrm{d} s$ for $t \in J$ and $\dot{x}^{*}(t)$ is Lebesgue integrable, $x^{*}(t)$ will be absolutely continuous and therefore $\left(x^{*}(\cdot), u^{*}(\cdot)\right)$ is a solution of problem (1). Thus, the proof is complete.

Introducing slack variable $v(t) \triangleq \dot{x}(t)$, problem (3) can be expressed as an optimal control problem as follows:

$$
\begin{aligned}
& \min \int_{J} F(x(t), v(t), u(t), t) \mathrm{d} t \\
& \text { s.t. } \\
& \dot{x}(t)=v(t) \\
& x(t) \in A, u(t) \in U, v(t) \in V \\
& x\left(t_{a}\right)=x_{a}, x\left(t_{b}\right)=x_{b}, t \in J
\end{aligned}
$$

where $A, U, V$, and $J$ are as before. It is easy to show that problems (3) and (4) are equivalent.

Consider $v(t)$ as a new control vector and define $\hat{u}(t) \triangleq\left[\begin{array}{l}u(t) \\ v(t)\end{array}\right]$. Therefore, problem (4) can be rewritten as:

$$
\begin{aligned}
& \min \int_{J} \hat{F}(x(t), \hat{u}(t), t) \mathrm{d} t \\
& \text { s.t. } \\
& \dot{x}(t)=\left[0_{n \times m}, \mathrm{I}_{n \times n}\right] \cdot \hat{u}(t) \\
& x(t) \in A, \hat{u}(t) \in \hat{U} \\
& x\left(t_{a}\right)=x_{a}, x\left(t_{b}\right)=x_{b}, t \in J
\end{aligned}
$$

where $\hat{F}(x(t), \hat{u}(t), t) \triangleq F(x(t), v(t), u(t), t)$ and $\hat{U} \triangleq U \times V \subseteq R^{n+m}$.

Definition 3.1. The trajectory-control pair $w=(x(\cdot), \hat{u}(\cdot))$ in problem $(5)$ is called admissible if the following conditions hold:

1) $x(\cdot)$ is absolutely continuous on $J$ and satisfies $x(t) \in A, t \in J$.
2) $\hat{u}(\cdot)$ is bounded Lebesgue measurable on $J$ and takes its values in $\hat{U}$.

3) Boundary conditions $x\left(t_{a}\right)=x_{a}$ and $x\left(t_{b}\right)=x_{b}$ are satisfied.

4) The state equation $\dot{x}(t)=\left[0_{n \times m}, \mathrm{I}_{n \times n}\right] \cdot \hat{u}(t)$ is satisfied.

Let $W$ be the set of all admissible pairs. Then, problem (5) (and equivalently (1), (3) and (4)) has a solution when $W$ is non-empty.

Some characteristics of the admissible pairs are as follows. Let $w=(x(\cdot), \hat{u}(\cdot))$ be an admissible pair, and $B$ be an open ball in $R^{n+1}$ containing $A \times J$. Let $C^{\prime}(B)$ be the space of all real-valued functions that are uniformly continuous on $B$ together with their first derivatives. Let $\phi \in C^{\prime}(B)$ and define function $\phi^{\vartheta}$ as follows:

$$
\begin{aligned}
& \phi^{\vartheta}(x(t), \hat{u}(t), t) \triangleq \phi_{x}(x(t), t) v(t)+\phi_{t}(x(t), t) \\
& \forall(x(t), \hat{u}(t), t) \in \Sigma, \forall \phi \in C^{\prime}(B)
\end{aligned}
$$

where $\Sigma=A \times \hat{U} \times J$. Also, $\phi^{9}$ is in the space $C(\Sigma)$, of real valued continuous functions defined on the compact set $\Sigma$. Since $w=(x(\cdot), \hat{u}(\cdot))$ is admissible pair, for all $\phi \in C^{\prime}(B)$ we have:

$$
\begin{aligned}
& \int_{J} \phi^{\vartheta}(x(t), \hat{u}(t), t) \mathrm{d} t \\
& =\int_{J}\left(\phi_{x}(x(t), t) v(t)+\phi_{t}(x(t), t)\right) \mathrm{d} t \\
& =\int_{J} \dot{\phi}(x(t), t) \mathrm{d} t=\phi\left(x_{b}, t_{b}\right)-\phi\left(x_{a}, t_{a}\right) \triangleq \Delta \phi
\end{aligned}
$$

Note that it is necessary to introduce the set $B$ and the space $C^{\prime}(B)$ since $A$ may have an empty interior in $R^{n}$. Now, consider $x_{j}(t)$ and $v_{j}(t)$ as the components of $x(t)$ and $v(t)$, respectively. Let $D\left(J^{\circ}\right)$ be the space of infinitely differentiable real-valued functions with compact support in $J^{\circ}=\left(t_{a}, t_{b}\right)$. Define:

$$
\begin{aligned}
& \psi_{j}(x(t), \hat{u}(t), t) \triangleq x_{j}(t) \dot{\psi}(t)+v_{j}(t) \psi(t) \\
& j=1,2, \cdots, n, \forall \psi \in D\left(J^{\circ}\right)
\end{aligned}
$$

Then, if $w=(x(\cdot), \hat{u}(\cdot))$ is an admissible pair, for $j=1,2, \cdots, n$ and $\psi \in D\left(J^{\circ}\right)$ we have:

$$
\begin{aligned}
& \int_{J} \psi_{j}(x(t), \hat{u}(t), t) \mathrm{d} t=\int_{J}\left(x_{j}(t) \dot{\psi}(t)+v_{j}(t) \psi(t)\right) \mathrm{d} t \\
& =\int_{J}\left(x_{j}(t) \dot{\psi}(t)+\dot{x}_{j}(t) \psi(t)-\dot{x}_{j}(t) \psi(t)+v_{j}(t) \psi(t)\right) \mathrm{d} t \\
& =\int_{J}\left(\frac{\mathrm{d}}{\mathrm{d} t}\left(x_{j}(t) \psi(t)\right)-\dot{x}_{j}(t) \psi(t)+v_{j}(t) \psi(t)\right) \mathrm{d} t \\
& =\left.x_{j}(t) \psi(t)\right|_{J}-\int_{J}\left(\left(\dot{x}_{j}(t)-v_{j}(t)\right) \psi(t)\right) \mathrm{d} t=0
\end{aligned}
$$


since the function $\psi$ has compact support in $J^{\circ}$, i.e. $\psi\left(t_{a}\right)=\psi\left(t_{b}\right)=0$, and the trajectory and control functions in an admissible pair satisfy the state equation in (5).

Now, consider a special choice of function $\phi$ in $C^{\prime}(B)$ which depends on the time variable only, i.e. $\phi(x(t), t)=\theta(t)$. In the light of (6) we have

$\phi^{\vartheta}(x(t), \hat{u}(t), t)=\dot{\theta}(t) \in C_{1}(\Sigma)$ where $C_{1}(\Sigma)$ is a sub-

space of $C(\Sigma)$ comprised of those continuous functions which depend only on the time variable. In addition, (7) implies that:

$$
\begin{aligned}
& \int_{J} \phi^{\vartheta}(x(t), \hat{u}(t), t) \mathrm{d} t=\int_{J} \dot{\theta}(t) \mathrm{d} t=\theta\left(t_{b}\right)-\theta\left(t_{a}\right) \\
& \forall \phi^{\vartheta} \in C_{1}(\Sigma)
\end{aligned}
$$

The set of Equalities (7), (9) and (10) are the properties of admissible pairs in the new but classical formulation of the path planning problem. In the next section, by suitable generalization, a transformation into another, non-classical problem is introduced which has better properties in some aspects.

\section{Metamorphosis}

For each admissible pair $w=(x(\cdot), \hat{u}(\cdot))$ consider the following well-defined mapping:

$$
\Lambda_{w}: H \in C(\Sigma) \rightarrow \int_{J} H(x(t), \hat{u}(t), t) \mathrm{d} t \in R^{+}
$$

This mapping is a bounded linear functional on $C(\Sigma)$ which is also positive, i.e. $\Lambda_{w}$ assigns nonnegative values to the nonnegative continuous functions $H$ on $\Sigma$.

Proposition 4.1. Let $\frac{\partial g}{\partial x}$ be continuous on $\Omega$ and $u(t)$ be piecewise continuous on $J$. The transformation $w \rightarrow \Lambda_{w}$ from $W$, the set of admissible pairs $w=(x(\cdot), \hat{u}(\cdot))$, into the space of bounded linear functional on $C(\Sigma)$ is an injection.

Proof. It must be shown that if $w_{1}=\left(x_{1}(\cdot), \hat{u}_{1}(\cdot)\right) \neq w_{2}=\left(x_{2}(\cdot), \hat{u}_{2}(\cdot)\right)$, then $\Lambda_{w_{1}} \neq \Lambda_{w_{2}}$. Indeed, if $w_{1} \neq w_{2}$, then by relation (5), $x_{1} \neq x_{2}$. Since $x_{1}$ and $x_{2}$ are continuous, there is a subinterval $J_{1} \in J$ such that $x_{1}(t) \neq x_{2}(t), \forall t \in J_{1}$. Now, a continuous function $H$ can be constructed such that it is equal zero for all $t$ outside $J_{1}$ and positive on the appropriate portion of the graph of $x_{1}(\cdot)$ and zero on that of $x_{2}(\cdot)$. Then, $\Lambda_{w_{1}}(H) \neq \Lambda_{w_{2}}(H)$, and the proof is complete.

Let $\Lambda$ be a linear positive continuous functional on $C(\Sigma)$. By Riesz representation Theorem, there exists a unique positive Radon measure $\mu$ on $\Sigma$ such that:

$$
\Lambda(H)=\int_{\Sigma} H \mathrm{~d} \mu \triangleq \mu(H), \forall H \in C(\Sigma)
$$

It is said that $\mu$ is a representing measure for $\Lambda$. Let
$M^{+}(\Sigma)$ be the space of all positive Radon measure on $\Sigma$. Then, solving problem (5) is equivalent to seek a measure in $M^{+}(\Sigma)$, denoted by $\mu^{*}$, which minimizes the functional:

$$
\mu \in M^{+}(\Sigma) \rightarrow \mu(\hat{F}) \in R^{+}
$$

over the set $Q_{w}$ of the measures $\mu$, corresponding to the admissible pairs $w=(x(\cdot), \hat{u}(\cdot))$, which satisfy:

$$
\begin{aligned}
& \mu\left(\phi^{\vartheta}\right)=\Delta \phi, \forall \phi \in C^{\prime}(B) \\
& \mu\left(\psi_{j}\right)=0, j=1,2, \cdots, n, \forall \psi \in D\left(J^{\circ}\right) \\
& \mu(f)=a_{f}, \forall f \in C_{1}(\Sigma)
\end{aligned}
$$

Existence of the optimal measure $\mu^{*}$ in the set $Q_{w}$ is equivalent to the controllability of problem (1). If $Q_{w}$ is compact, then existing of the optimal measure $\mu^{*}$ is guaranteed, as the map $\mu \rightarrow \mu(\hat{F}), \mu \in Q_{w}$, mapping $Q_{w}$ into the real line, is continuous, where $Q_{w}$ is considered to have relative topology induced by the topology of $M^{+}(\Sigma)$. But in general $Q_{w}$ may not be compact. However, if we extend $Q_{w}$ to the set $Q$ including all measures in $M^{+}(\Sigma)$ which satisfy (14) (not necessarily those measures corresponding to the admissible pairs), then the optimal measure $\mu^{*}$ in $Q$ exists which is shown by the following theorem:

Theorem 4.1. Let $Q \subseteq M^{+}(\Sigma)$ be the set of all positive Radon measure on $\Sigma$ satisfying (14). There exists an optimal measure $\mu^{*}$ in the set $Q$ for which

$\mu^{*}(\hat{F}) \leq \mu(\hat{F})$, for all $\mu \in Q$.

Proof. It is similar to the proof of Theorem II.1 in [20] and we neglect it.

Notice that in this case, which the set $Q_{w}$ is extended to the set $Q, \mu^{*}$ is not necessarily a measure corresponding to an admissible pair $w$.

Remark 4.1. Minimization of functional (13) subject to (14) is an infinite-dimensional LP. But it is possible to approximate the solution of this problem by the solution of a finite-dimensional LP of sufficiently large dimension which will be done in the next section.

\section{Approximation}

For the first step of approximation, we consider the measures in $M^{+}(\Sigma)$ satisfying a finite number of constraints in (14). To do this, let $\left\{\phi_{k}: k=1,2, \cdots, M_{1}\right\}$ and $\left\{\chi_{h}: h=1,2, \cdots, M_{2}\right\}$ be subsets of some total sets in $C^{\prime}(B)$ and $D\left(J^{\circ}\right)$, respectively (A subset of $C^{\prime}(B)$ or $D\left(J^{\circ}\right)$ is total if the linear combinations of its elements be dense in that space).

Theorem 5.1. Consider LP consisting of minimizing the function $\mu \rightarrow \mu(\hat{F})$ over the set $Q\left(M_{1}, M_{2}\right)$ 
which is the set of all measures in $M^{+}(\Sigma)$ satisfying:

$$
\begin{aligned}
& \mu\left(\phi_{k}^{9}\right)=\Delta \phi_{k}, k=1,2, \cdots, M_{1} \\
& \mu\left(\chi_{h}\right)=0, h=1,2, \cdots, M_{2}
\end{aligned}
$$

Then, as $M_{1}$ and $M_{2}$ tend to the infinity,

$$
\begin{aligned}
& \mu\left(M_{1}, M_{2}\right)=\inf _{\mu \in Q\left(M_{1}, M_{2}\right)} \mu(\hat{F}) \text { tends to } \\
& \mu^{*}(\hat{F})=\inf _{\mu \in Q} \mu(\hat{F}) .
\end{aligned}
$$

Proof. It is similar to the proof of Theorem III.1 in [20]. The above theorem provides the theoretical justifications to approximate the infinite number of constraints by a finite number of them. Now, consider the problem of minimizing (13) over $Q\left(M_{1}, M_{2}\right)$. By Theorem A.5 in [20], the optimal measure of this problem has the following form:

$$
\mu^{*}=\sum_{j=1}^{M_{1}+M_{2}} \alpha_{j}^{*} \delta\left(z_{j}^{*}\right)
$$

where $\alpha_{j}^{*} \geq 0, z_{j}^{*} \in \Sigma$, and $\delta(z)$ is the unitary atomic measure characterized by:

$$
\delta(z)(H)=H(z), H \in C(\Sigma), z \in \Sigma
$$

The above representation of $\mu^{*}$ as a combination of unitary atomic measures changes the strange problem of finding a measure in the set $Q\left(M_{1}, M_{2}\right)$ to a problem of finding $\left\{\left(\alpha_{j}^{*}, z_{j}^{*}\right): \alpha_{j}^{*} \in R^{+}, z_{j}^{*} \in \Sigma, j=1,2, \cdots, M_{1}+M_{2}\right\}$. If we could reduce this problem to the one in which $z_{1}^{*}, z_{2}^{*}, \cdots, z_{M_{1}+M_{2}}^{*}$ are fixed, and unknowns be the non-negative coefficients $\alpha_{1}^{*}, \alpha_{2}^{*}, \cdots, \alpha_{M_{1}+M_{2}}^{*}$, then we would have a finite dimensional LP. This is the second stage of approximation. We can approximate the optimal measure with:

$$
\mu^{*} \cong \sum_{j=1}^{N} \alpha_{j}^{*} \delta\left(z_{j}\right)
$$

where $N \gg M_{1}+M_{2}$ and $z_{1}, z_{2}, \cdots, z_{N}$ are fixed in a countable dense subset of $\Sigma$ [20]. Therefore, the finite-dimensional LP problem is:

$$
\begin{aligned}
& \min \sum_{j=1}^{N} \alpha_{j} \hat{F}\left(z_{j}\right) \\
& \text { s.t. } \\
& \sum_{j=1}^{N} \alpha_{j} \phi_{k}^{9}\left(z_{j}\right)=\Delta \phi_{k}, k=1,2, \cdots, M_{1} \\
& \sum_{j=1}^{N} \alpha_{j} \chi_{h}\left(z_{j}\right)=0, h=1,2, \cdots, M_{2} \\
& \sum_{j=1}^{N} \alpha_{j} f_{s}\left(z_{j}\right)=a_{s}, s=1,2, \cdots, L \\
& \alpha_{j} \geq 0, j=1,2, \cdots, N
\end{aligned}
$$

The function $f_{s}$ in (19) depends on the time only and is chosen as piecewise constant function as follows:

$$
f_{s}(t)=\left\{\begin{array}{ll}
1 & \text { if } t \in J_{s} \\
0 & \text { otherwise }
\end{array} \quad s=1,2, \cdots, L\right.
$$

where $J_{s}=\left(t_{a}+(s-1) d, t_{a}+s d\right)$ and $d=\frac{\Delta t}{L}=\frac{t_{b}-t_{a}}{L}$.

Remark 5.1. Note that $f_{s}$ should be in $C_{1}(\Sigma)$, however in order to avoid the infeasibility of LP problem (19), we have chosen the Walsh functions instead, by the fact that that every continuous function in $C_{1}(\Sigma)$ can be approximated by a linear combination of these functions. This is, in fact, another step of approximation.

Now, by solving LP (19), optimal values of decision variables $\left\{\alpha_{1}, \alpha_{2}, \cdots, \alpha_{N}\right\}$ are found. The procedure to construct a piecewise constant control function approximating the action of optimal measure is based on the analysis in [20]. Here, we proceed by this approach and construct $\hat{u}(t)$ and $u(t)$ as piecewise constant functions. The state trajectory $x(t)$ is also obtained as the response of nonlinear system in (1) with $x\left(t_{a}\right)=x_{a}$ to the control $u(t)$. As it has been proved in [20], when $N, M \rightarrow \infty$, the obtaind $u(t)$ and $x(t)$ tend to the exact control function and state trajectory, respectively, in a way that the initial condition $x\left(t_{a}\right)=x_{a}$ is always satisfied and the final condition is going to be satisfied, i.e. $x\left(t_{b}\right) \rightarrow x_{b}$ as $N, M \rightarrow \infty$.

Remark 5.2. Referring to Theorem 3.1., the optimal value of objective function in (19) can be considered as a criterion for the total error. After solving (19) if the total error is more than desirable one, it can be improved by increasing the number of variables $N$ or constraints $M$, of the LP problem (19). Therefore, in this approach the accuracy can be improved as fine as desired.

\section{Numerical Example}

In this section we present a numerical example to show the effectiveness of the proposed approach for solving nonlinear path planning problems with a systematic algorithm. Thus, consider the following problem which has too complicated nonlinear term [19]:

$$
\begin{aligned}
& \dot{x}=0.5 x^{2}(t) \sin (x(t))+u(t) \\
& \text { s.t. } \\
& x(t) \in[0,1], u(t) \in[0,1] \\
& x(0)=0, x(1)=0.5 \\
& J=[0,1]
\end{aligned}
$$

Step 1. Let $\varepsilon=10^{-3}$ as permitted error and divide each of $J, A, V$, and $U$ into 10 parts. Thus, 
$N=10 \times 10 \times 10 \times 10=10^{4}$.

Step 2. Let $M_{1}=2, M_{2}=8, L=10$ and determine $\phi_{k}, \chi_{h}$, and $f_{s}$ as follows:

$$
\begin{aligned}
& \left\{\begin{array}{l}
\phi_{k}=x_{i}^{j} \\
k=1,2, \cdots, M_{1}, i=1,2, \cdots, n, j=1,2, \cdots, \frac{M_{1}}{n}
\end{array}\right. \\
& \phi_{k}^{\vartheta}=\frac{\partial \phi_{k}}{\partial x} v(t), k=1,2, \cdots, M_{1} \\
& \psi_{r, 1}(t)=\sin \left(\frac{2 \pi r\left(t-t_{a}\right)}{\Delta t}\right) \\
& \left\{\psi_{r, 2}(t)=1-\cos 2 \pi\left(\frac{2 \pi r\left(t-t_{a}\right)}{\Delta t}\right)\right. \\
& r=1,2, \cdots, \frac{M_{2}}{2 n}, \Delta t=t_{b}-t_{a}=1-0=1 \\
& \left\{\begin{array}{l}
\chi_{h}=x_{j}(t) \dot{\psi}_{r, 1}(t)+v_{j}(t) \psi_{r, 1}(t) \\
h=1,2, \cdots, \frac{M_{2}}{2}, j=1,2, \cdots, n, r=1,2, \cdots, \frac{M_{2}}{2 n}
\end{array}\right. \\
& \left\{\begin{array}{l}
\chi_{h}=x_{j}(t) \dot{\psi}_{r, 2}(t)+v_{j}(t) \psi_{r, 2}(t) \\
h=\frac{M_{2}}{2}+1, \frac{M_{2}}{2}+2, \cdots, M_{2} \\
j=1,2, \cdots, n \\
r=1,2, \cdots, \frac{M_{2}}{2 n}
\end{array}\right. \\
& f_{s}= \begin{cases}1 & z \in J_{s} \\
0 & \text { otherwise }\end{cases} \\
& J_{s}=\left(\frac{s-1}{10}, \frac{s}{10}\right), s=1,2, \cdots, 10
\end{aligned}
$$

Thus, $a_{s}=0.1$ for $s=1,2, \cdots, 10$ as the integral of $f_{s}$ over $J_{s}$.

Step 3. Solve the LP (19) with $N=10^{4}$ variables and $M=M_{1}+M_{2}+L=2+8+10=20$ constraints.

Step 4. Calculate $\hat{u}(t)$ and then apply $u(t)$ to the nonlinear system in (21) with $x(0)=0$ to obtain $x(t)$. Figures 1 and 2 illustrate the results.

Simulation results show that the constraints $x(t) \in[0,1]$ and $u(t) \in[0,1]$ are satisfied, and the nonlinear control system is steered from the known initial state $x(0)=0$ to the desired final state $x(1)=0.5$ in the certain finite time interval $J=[0,1]$.

Step 5. Compare the total error with desirable one. In this example, after solving LP the total error would be $e=1.784 \times 10^{-6}$ as the optimal value of objective function in LP. Since $e \ll \varepsilon=10^{-3}$ it can be said that path planning problem has been solved approximately with the

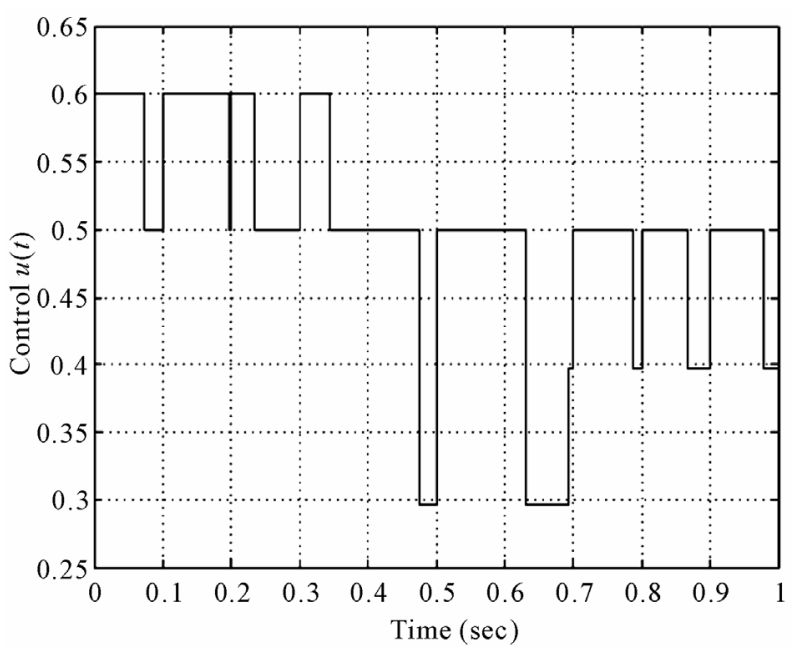

Figure 1. Control function $u(t)$.

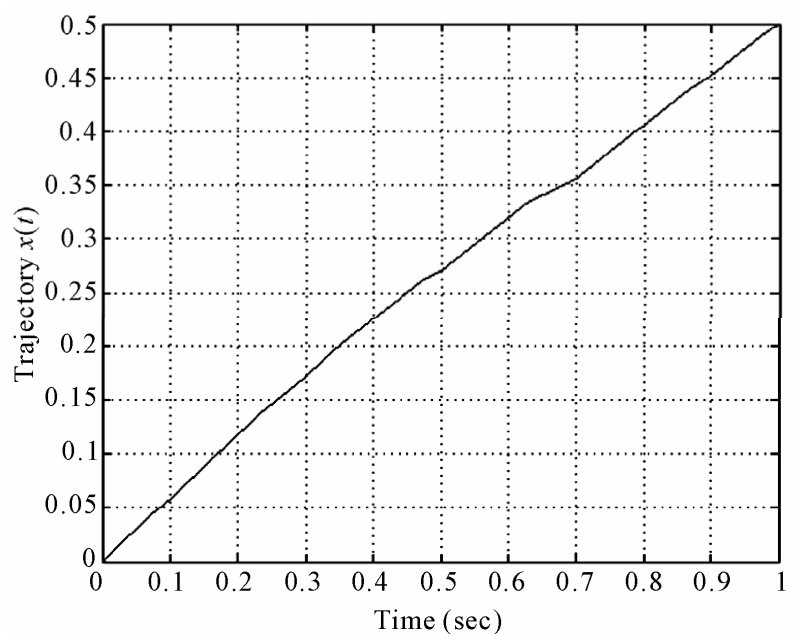

Figure 2. State trajectory $x(t)$.

total error $e=1.784 \times 10^{-6}$.

Problem (21) has also been solved approximately in [19] by solving a sequence of NLP problems. The optimal value of objective function has been obtained as $4.6089 \times 10^{-5}$. Therefore, our proposed approach, in comparison with what has been proposed in [19], has more accuracy together with lower computational load.

\section{Conclusions}

In this paper, a new approach has been proposed to find an approximate solution for the nonlinear path planning problem. In this approach, first a new problem, equivalent to the original problem, has been defined in the calculus of variations. The new problem can be expressed as an optimal control problem by introducing slack variable. Then, a measure theoretical approach and two stage approximations have been used to convert the optimal 
control problem to a finite dimensional LP. The solution of this LP is used to construct an approximate solution for the original path planning problem. The proposed approach in comparison with other numerical methods works well; especially it is practical and accurate enough for systems with too complicated nonlinear terms. Moreover, error is completely controllable and accuracy can be improved as fine as desired. In addition, as the obtained control function is piecewise constant, it is suitable for switching systems. Effectiveness of the proposed approach has been verified using a numerical example.

\section{Acknowledgements}

The authors gratefully acknowledge the helpful comments and suggestions of the reviewers, which have improved the manuscript.

\section{References}

[1] J. M. Athans and P. L. Falb, "Optimal Control: An Introduction to the Theory and Its Applications," McGrawHill, New York, 1996.

[2] A. T. Hasan, A. M. S. Hamouda, N. Ismail and H. M. A. A. Al-Assadi, "A New Adaptive Learning Algorithm for Robot Manipulator Control," Proceedings of the Institution of Mechanical Engineers Part I-Journal of Systems and Control Engineering, Vol. 221, No. 4, 2007, pp. 663-672. doi:10.1243/09596518JSCE321

[3] A. S. Rana and A. M. S. Zalzala, "Collision-Free Motion Planning of Multi-Arm Robots Using Evolutionary Algorithms," Proceedings of the Institution of Mechanical Engineers Part I-Journal of Systems and Control Engineering, Vol. 211, No. 5, 1997, pp. 373-384. doi:10.1243/0959651971539902

[4] C. L. Chen and C. J. Lin, "Motion Planning of Redundant Robot Manipulators Using Constrained Optimization: A Parallel Approach," Proceedings of the Institution of Mechanical Engineers Part I-Journal of Systems and Control Engineering, Vol. 212, No. 4, 1998, pp. 281-292. doi:10.1243/0959651981539460

[5] Y. Wang, D. M. Lane and G. J. Falconer, "Two Novel Approaches for Unmanned under Water Vehicle Path Planning: Constrained Optimization and Semi-Infinite Constrained Optimization," Robotica, Vol. 18, No. 2, 2000, pp. 123-142. doi:10.1017/S0263574799002015

[6] J. C. Latombe, "Robot Motion Planning," Kluwer Academic Publishers, Boston, 1991.

[7] L. Kavarki, P. Svestka, J. Latombe and M. Overmars, "Probabilistic Road Maps for Path Planning in High Dimensional Configuration Space," IEEE Transactions on Robotics and Automation, Vol. 12, No. 4, 1996, pp. 566-580. doi:10.1109/70.508439
[8] G. C. Luh and W. W. Liu, "Motion Planning for Mobile Robots in Dynamic Environments Using a Potential Field Immune Network," Proceedings of the Institution of Mechanical Engineers Part I-Journal of Systems and Control Engineering, Vol. 221, No. 7, 2007, pp. 1033-1045. doi:10.1243/09596518JSCE400

[9] L. Kavarki, M. Kolountzakis and J. Latombe, "Analysis of Probabilistic Road Maps for Path Planning," IEEE Transactions on Robotics and Automation, Vol. 14, No. 1, 1998, pp. 166- 171. doi:10.1109/70.660866

[10] Y. Koren and J. Borenstein, "Potential Field Methods and Their Inherent Limitations for Mobile Robot Navigation," Proceedings of the IEEE Conference on Robotics and Automation, Sacramento, 1991. doi:10.1109/ROBOT.1991.131810

[11] P. C. Zhou, B. R. Hong and J. H. Yang, "Chaos Genetic Algorithm Based Path Planning Method for Mobile Robot," Journal of Harbin Institute of Technology, Vol. 36, No. 7, 2004, pp. 880-883.

[12] Y. O. Qin, D. B. Sun, N. Li and Y. G. Cen, "Path Planning for Mobile Robot Using the Particle Swarm Optimization with Mutation Operator," Proceedings of the 3rd International Conference on Machine Learning and Cybernetics, Shanghai, 2004.

[13] T. Cecil and D. E. Marthaler, "A Variational Approach to Path Planning in Three Dimensions Using Level Set Methods," Journal of Computational Physics, Vol. 211, No. 1, 2006, pp. 179-197. doi:10.1016/j.jcp.2005.05.015

[14] M. G. Earl and R. Danderia, "Modelling and Control of a Multi-Agent System Using Mixed Integer Linear Programming," Proceedings of the 41st IEEE Conference on Decision and Control, Las Vegas, 10-13 December 2002. doi:10.1109/CDC.2002.1184476

[15] A. Richards and J. How, "Aircraft Trajectory Planning with Collision Avoidance Using Mixed Integer Programming," Proceedings of the IEEE American Control Conference, Anchorage, Alaska, 2002.

[16] M. Gachpazan and A. V. Kamyad, "Solving of Second Order Nonlinear PDE Problems by Using Artificial Controls with Controlled Error," Korean Journal of Computational \& Applied Mathematics, Vol. 15, No. 1-2, 2004, pp. 173-184.

[17] S. A. Alavi, A. V. Kamyad and M. Gachpazan, "Solving of Nonlinear Ordinary Differential Equations as a Control Problem by Using Measure Theory," Scientia Iranica, Vol. 7, No. 1, 2000, pp. 1-7.

[18] M. Gachpazan, A. Kerayechian and A. V. Kamyad, "A New Method for Solving Nonlinear Second Order Differential Equations," Korean Journal of Computational \& Applied Mathematics, Vol. 7, No. 2, 2000, pp. 333-345.

[19] A. Jajarmi, M. Gachpazan and A. V. Kamyad, "Openloop Control of Nonlinear Systems via a Sequence of Nonlinear Programming Problems," Proceedings of the 17th Iranian Conference of Electrical Engineering, Tehran, 13-15 August 2009.

[20] J. E. Rubio, "Control and Optimization, the Linear 
Treatment of Non-linear Problems," Manchester University Press, Manchester, 1986.

[21] A. V. Kamyad, J. E. Rubio and D. A. Wilson, "An Optimal Control Problem for the Multidimensional Diffusion Equation with a Generalized Control Variable," Journal of Optimization Theory and Applications, Vol. 75, No. 1, 1992, pp. 101-132. doi:10.1007/BF00939908

[22] A. V. Kamyad and A. H. Borzabadi, "Strong Controllability and Optimal Control of the Heat Equation with a Thermal Source," Korean Journal of Computational \& Applied Mathematics, Vol. 7, No. 3, 2002, pp. 555-568.
[23] A. H. Borzabadi, A. V. Kamyad and M. H. Farahi, “Optimal Control of the Heat Equation in an Inhomogeneous Body," Applied Mathematics and Computation, Vol. 15, No. 1-2, 2004, pp. 127-146.

[24] D. Hinrichsen and A. J. Pritchard, "Mathematical System Theory I: Modeling, State Space Analysis, Stability and Robustness," Springer, Berlin, 2005.

[25] A. V. Kamyad and H. H. Mehneh, "A Linear Programming Approach to the Controllability of Time-Varying Systems," International Journal of Engineering Science, Vol. 14, No. 8, 2003, pp. 143-151. 